\title{
Custo-efetividade das abordagens terapêuticas da leucemia linfocítica aguda em pacientes pediátricos
}

\author{
Cost-effectiveness of therapeutic approaches to acute lymphocytic leukemia in pediatric patients \\ Coste-efectividad de los enfoques terapéuticos para la leucemia linfocítica aguda en pacientes \\ pediátricos
}

Recebido: 20/10/2021 | Revisado: 27/10/2021 | Aceito: 03/11/2021 | Publicado: 06/11/2021

\author{
Francisca Thaís de Sousa Cavalcante \\ ORCID: https://orcid.org/0000-0002-8386-4618 \\ Universidade Federal da Paraíba, Brasil \\ E-mail: thaissousa.cavalcante@ hotmail.com \\ Thayana Maria Navarro Ribeiro de Lima \\ ORCID: https://orcid.org/0000-0003-2881-4818 \\ Universidade Federal da Paraíba, Brasil \\ E-mail: thayana.maria.navarro@gmail.com \\ Paula Maria Maracajá Bezerra \\ ORCID: https://orcid.org/0000-0002-9705-8959 \\ Universidade Federal da Paraíba, Brasil \\ E-mail: paulammaracaja@gmail.com \\ Thiago Isidro Vieira \\ ORCID: https://orcid.org/0000-0003-4824-9131 \\ Universidade Federal da Paraíba, Brasil \\ E-mail: thiago_isidro@yahoo.com.br \\ Yuri Wanderley Cavalcanti \\ ORCID: https://orcid.org/0000-0002-3570-9904 \\ Universidade Federal da Paraíba, Brasil \\ E-mail: yuri.wanderley@yahoo.com.br \\ Simone Alves de Sousa \\ ORCID: https://orcid.org/0000-0002-3254-9036 \\ Universidade Federal da Paraíba, Brasil \\ E-mail: simonealvess.sousa@gmail.com
}

\begin{abstract}
Resumo
Objetivo: Realizar uma análise de custo-efetividade dos protocolos antineoplásicos mais utilizados no tratamento da leucemia aguda em pacientes pediátricos. Métodos: Realizou-se uma análise de custo-efetividade, com uso de modelo de árvore de decisão, a partir da consulta de dados secundários de sistemas de informação em saúde do Brasil, o SIGTAP - SUS. A população foi composta por pacientes pediátricos registrados na base de dados do RHC do INCA, na faixaetária de 0 a 19 anos, apresentando leucemia linfocítica/linfoide/linfoblástica como neoplasia de base, no período de 1997-2017. Resultados: Para os pacientes do sexo masculino, o transplante de medula óssea (TMO) exibiu um custo incremental alto em relação às alternativas e demonstrou a menor efetividade. $O$ tratamento realizado com $Q t$ associada ao TMO (QtTMO), embora de custo incremental maior, apresentou efetividade muito superior às alternativas, tornandose custo-efetiva. A associação de Qt com radioterapia (QtRxt) se revelou o tratamento com maior custo-efetividade. Para o sexo feminino, o tratamento realizado com QtTMO se apresentou como o menos custo-efetivo, juntamente com o TMO. Por sua vez, a QtRxt se mostrou com custo-efetividade maior em relação às alternativas. Conclusão: Os resultados desta análise econômica revelaram melhor custo-efetividade para a QtRxt e pior custo-efetividade para a TMO, em ambos os sexos. Essa análise mostrou diferentes cenários de acordo com o sexo do paciente, em particular, o tratamento com QtTMO pode constituir uma opção terapêutica custo-efetiva para os pacientes do sexo masculino, porém não para os do sexo feminino.
\end{abstract}

Palavras-chave: Saúde da criança; Leucemia linfoide; Análise custo-benefício.

\begin{abstract}
Objective: To carry out a cost-effectiveness analysis of the most used antineoplastic treatment protocols of acute leukemia in pediatric patients. Methods: Conducted a cost-effectiveness analysis, using the decision tree model, based on the consultation of secondary data from health information systems in Brazil, SIGTAP - SUS. The population consisted of pediatric patients registered in RHC database of INCA, aged 0 to 19 years, showing lymphocytic / lymphoid / lymphoblastic leukemia as a neoplasm basis, in a period from 1997 to 2017. Results: For male patients, bone marrow
\end{abstract}


transplantation (BMT) exhibited a high increase in the ratio as alternatives and demonstrated the least effectiveness. Despite high incremental costs, the treatment performed with $\mathrm{Ct}$ associated with BMT (CtMT), showed effectivess much higher than other alternatives, becoming a cost-effective treatment. The association of $\mathrm{Ct}$ with radiotherapy (CtRxt) revealed the most effective treatment. For females patients, the treatment performed with CtBMT has shown to be the least cost-effectiveness, along with BMT. In turn, CtRxt provides to be the best cost-effectiveness treatment. Conclusion: The results of this economic analysis revealed better cost-effectiveness for CtRxt and worse costeffectiveness for BMT, in both sexes. This analysis showed different scenarios according to the patient's gender, in particular, treatment with CtBMT can be an effective therapeutic option for male patients, but not for female patients.

Keywords: Child health; Leukemia, lymphoid; Cost-benefit analysis.

\section{Resumen}

Objetivo: Realizar un análisis de coste-efectividad de los protocolos antineoplásicos más utilizados en el tratamiento de la leucemia aguda en pacientes pediátricos. Métodos: Se realizó un análisis de costo-efectividad, utilizando un modelo de árbol de decisión, basado en la consulta de datos secundarios de los sistemas de información en salud de Brasil, el SIGTAP - SUS. La población estuvo constituida por pacientes pediátricos registrados en la base de datos de la RHC del INCA, de 0 a 19 años, con leucemia linfocítica / linfoide / linfoblástica como neoplasia basal en el período 1997-2017. Resultados: Para los pacientes masculinos, el trasplante de médula ósea (TMO) exhibió un alto costo incremental en comparación con las alternativas y demostró la menor efectividad. El tratamiento realizado con Qt asociado a TMO (QtBMT), aunque con un coste incremental mayor, resultó mucho más efectivo que otras alternativas, volviéndose rentable. La asociación de Qt con radioterapia (QtRxt) resultó ser el tratamiento más rentable. Para las mujeres, el tratamiento realizado con QtTMO demostró ser el menos rentable, junto con el BMT. A su vez, QtRxt demostró ser más rentable en comparación con otras alternativas. Conclusión: Los resultados de este análisis económico revelaron mejor costo-efectividad para QtRxt y peor costo-efectividad para BMT, en ambos sexos. Este análisis mostró diferentes escenarios según el sexo del paciente, en particular, el tratamiento con QtTMO puede ser una opción terapéutica rentable para pacientes masculinos, pero no para pacientes femeninas.

Palabras clave: Salud del niño; Leucemia linfoide; Análisis costo-beneficio.

\section{Introdução}

O câncer em crianças e adolescentes é considerado raro quando comparado ao câncer em adultos, correspondendo a 2$3 \%$ de todos os tumores malignos registrados no Brasil, embora já seja a primeira causa de óbitos por doença no país (INCA, 2016; INCA, 2020). Dentre essas neoplasias, a leucemia é a mais prevalente, representando cerca de um terço de todos os cânceres que afetam pacientes antes dos 15 anos de idade, obtendo uma prevalência de 25-35\% (Metayer, 2013; INCA, 2016).

A prevalência desse tipo de câncer, caracterizado pela proliferação anormal de precursores de glóbulos brancos, é maior para o sexo masculino (Santos, 2017). Dados recentes sobre o número de novos casos de leucemia esperados para o Brasil em 2020-2022 é de 5.920 casos em homens e de 4.890 em mulheres (INCA,2019). Dentre os principais tipos de leucemia, estão a leucemia mielóide aguda (LMA), a leucemia mielóide crônica (LMC), a leucemia linfocítica aguda (LLA) e leucemia linfocítica crônica (LLC). Dentre elas, a LLA representa a que mais acomete pacientes infantis, com prevalência de $80 \%$ em crianças abaixo dos 5 anos (INCA, 2019).

Atualmente, entende-se que a principal abordagem ao câncer infantil deve ser o diagnóstico precoce e encaminhamento para um tratamento oportuno e de qualidade, que possibilite maiores taxas de cura (Brasil, 2017). A escolha do método mais efetivo de tratamento da LLA deve considerar a condição clínica, imunológica e citogenética do paciente, bem como o comprometimento e/ou abrangência de outros órgãos. O tratamento para esse tipo de neoplasia é fundamentado na quimioterapia (Qt), e caso desenvolva sintomas negativos ou recaída da patologia deve-se abordar tratamentos mais agressivos, como o transplante de medula óssea (TMO) (Cavalcante, Rosa, Torres, 2017). O TMO associada à Qt é provavelmente a abordagem mais efetiva, entretanto, o sistema de saúde enfrenta dificuldade devido a escassez de doadores e alto custo do tratamento (Arnaldi, 2019).

Provavelmente devido à inclusão de novas terapias, drogas e tecnologias de saúde nas últimas décadas, houve uma evolução no tratamento da LLA, e como resultado, a taxa de sobrevivência aproxima-se de 90\% em muitos países desenvolvidos (Viana et al. 2015). Em consequência, também houve considerável aumento no custo de insumos e serviços (Arnaldi, 2019). 
Os estudos de avaliação econômica são adotados a fim de considerar o fator custo na tomada de decisão, uma vez que os recursos financeiros são escassos e finitos (Moraz, Garcez, Assis, 2015). Um dessas análises é a relação de custo-efetividade, que visa determinar a relação entre os custos e os benefícios decorrentes de intervenções. É o método mais indicado para se comparar duas ou mais alternativas terapêuticas, diagnósticas ou preventivas, por permitir a análise combinada de benefícios clínicos e os custos associados (Moraz, Garcez, Assis, 2015). Nos dias de hoje, a informação, com base em dados válidos, confiáveis e atualizados, é considerada o principal ativo da sociedade. Ter a informação correta, no momento oportuno, faz a diferença para um planejamento estruturado e coerente com a realidade, permitindo ações eficazes e efetivas (INCA, 2019).

A definição do tratamento das leucemias agudas precisa considerar também o custo para a gestão do serviço oncológico, a fim de garantir a manutenção dos resultados frente à cura e sobrevida conquistadas. Em todo o mundo, os custos dos tratamentos de cânceres vêm aumentando conforme crescimento do número de casos, sendo investimentos em pesquisas, diagnóstico precoce e diferentes modalidades de tratamento necessários. Portanto, torna-se importante desenvolver estudos econômicos levando em consideração os custos e os resultados das terapias antineoplásicas, já que pesquisas de avaliação econômica representam alternativas que integram dados monetários e de efetividade em um modelo para análise e tomada de decisão (Silva, 2019; Angevine, Berven, 2014).

Diante disso, o presente estudo tem como objetivo avaliar o custo-efetividade do tratamento da LLA em pacientes pediátricos.

\section{Metodologia}

\subsection{Delineamento da pesquisa}

\subsubsection{Desenho do estudo}

Trata-se de uma avaliação econômica completa do tipo custo-efetividade baseada em modelagem matemática e delineada de acordo com as diretrizes do Sistema de Gerenciamento da Tabela de Procedimentos e Órteses, Próteses e Materiais Especiais (OPM) - (SIGTAP) do Sistema Único de Saúde (SUS).

\subsubsection{População alvo}

A população para realização desse modelo foi composta por pacientes pediátricos registrados na base de dados do Registro Hospitalar de Câncer (RHC) do INCA, na faixa-etária de 0 a 19 anos, apresentando leucemia linfocítica/linfóide/linfoblástica como neoplasia de base, no período de 1997 a 2017.

\subsubsection{Análise da efetividade}

A efetividade foi mensurada através da razão de prevalência de não morrer. Tomou-se como referência a taxa de óbitos entre os indivíduos que se submeteram à quimioterapia. A razão de prevalência de não morrer foi estabelecida como o inverso da razão de prevalência de morrer.

\subsubsection{Custos}

Para a elaboração do cálculo, foram utilizados os custos diretos ambulatoriais, hospitalares e médicos para cada tipo de tratamento encontrado no SIGTAP do SUS. Os custos incluídos foram: quimioterapia ( $\mathrm{R} \$ 11.644,00)$; radioterapia $(\mathrm{R} \$ 3.159,00)$ e transplante de medula óssea (TMO). Para este último, foi estabelecida a média dos valores entre os custos do tratamento com doadores aparentados $(\mathrm{R} \$ 54.939,27)$ e não aparentados ( $\mathrm{R} \$ 71.602,25)$, resultando em $\mathrm{R} \$ 63.270,76$ como custo da TMO. Os valores dos tratamentos associados, como a quimioterapia com radioterapia (QtRxt) e a quimioterapia com TMO (QtTMO), são encontrados realizando-se a soma dos tratamentos individuais (Tabela 1). 


\subsection{Procedimento de análise dos dados}

\subsubsection{Análise de custo-efetividade}

O modelo escolhido para a análise dos dados foi a árvore de decisão, pois é a forma mais simples de representação dos eventos e suas respectivas probabilidades de ocorrência. São representados, graficamente, possíveis caminhos que os pacientes percorreriam caso estivessem sob as estratégias ou intervenções investigadas, podendo-se atribuir custos e desfechos em saúde a cada itinerário percorrido pelos indivíduos (Silva, Silva, Pereira, 2016). Os dados foram analisados no Software TreeAge para essa representação, como vistos nas Figuras 1 e 2.

Figura 1 - Árvore de decisão para o sexo masculino.

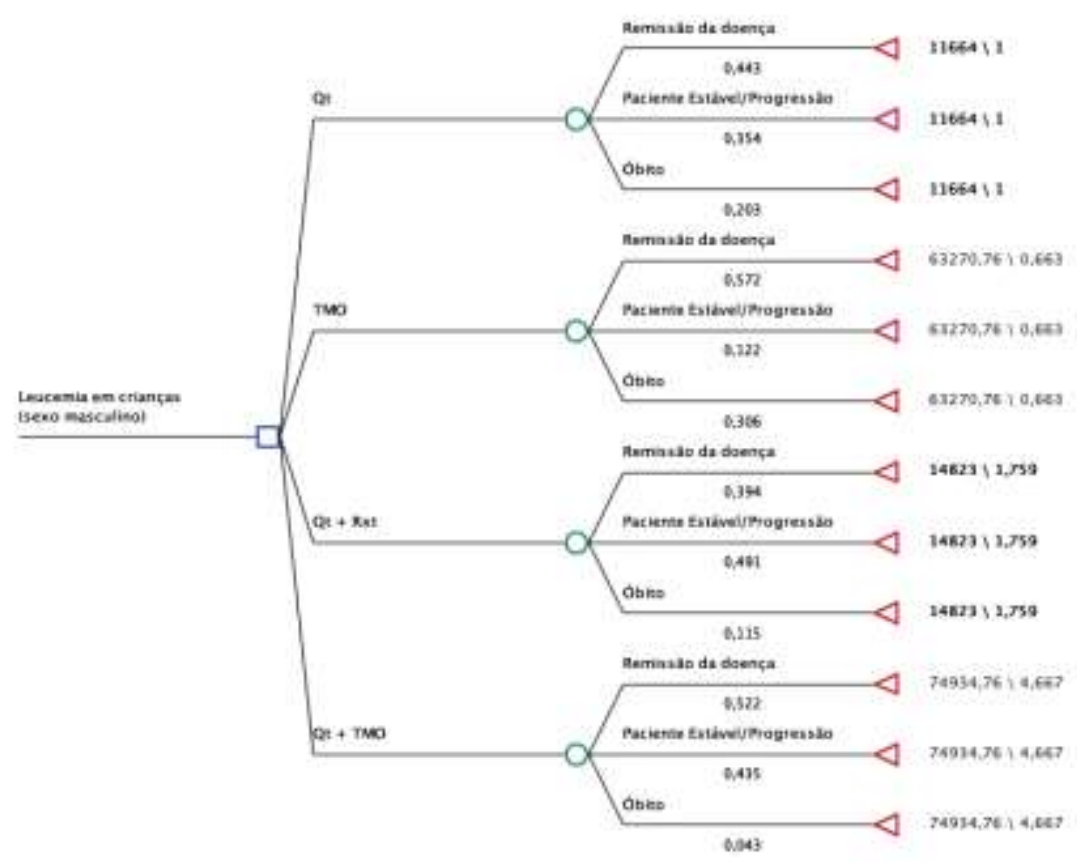

Fonte: Software TreeAge. 
Figura 2 - Árvore de decisão para o sexo feminino.

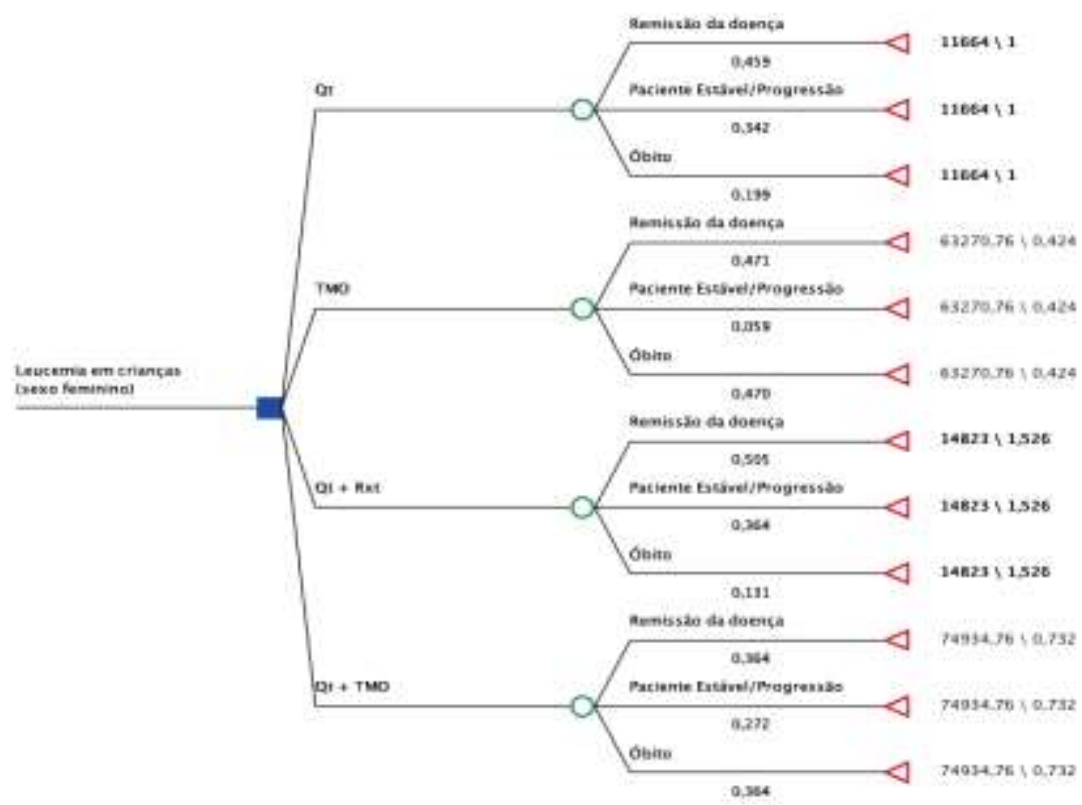

Fonte: Software TreeAge.

Dentre os tratamentos recebidos pelos pacientes, optou-se por inserir na análise a quimioterapia (Qt), o TMO, a quimioterapia associada à radioterapia (QtRxt) e a quimioterapia associada ao $\mathrm{TMO}(\mathrm{QtTMO})$, pois apresentaram um número maior de casos, tornando-se mais relevantes para o estudo.

\section{Resultados}

A Tabela 1 apresenta a distribuição dos pacientes de acordo com o sexo e o seu desfecho clínico.

Tabela 1 - Distribuição dos tipos de tratamento e respectivos custos, de acordo com o sexo dos pacientes e o desfecho clínico.

\begin{tabular}{c|ccccccc}
\hline \multirow{2}{*}{ Tratamento } & \multicolumn{6}{|c}{ Desfecho clínico } & \multirow{2}{*}{ Custos } \\
\cline { 2 - 7 } & Doença presente & \multicolumn{2}{|c}{ Óbito } & Doença em remissão & \\
\cline { 2 - 7 } & $\mathbf{M}$ & $\mathbf{F}$ & $\mathbf{M}$ & $\mathbf{F}$ & $\mathbf{M}$ & $\mathbf{F}$ & \\
\hline Qt & 1410 & 1007 & 808 & 589 & 1764 & 1353 & $\mathrm{R} \$ 11.644,00$ \\
TMO & 6 & 1 & 15 & 8 & 28 & 8 & $\mathrm{R} \$ 63.270,76$ \\
QtRxt & 102 & 39 & 24 & 14 & 82 & 54 & $\mathrm{R} \$ 14.803,00$ \\
QtTMO & 10 & 4 & 1 & 3 & 12 & 4 & $\mathrm{R} \$ 74.934,76$ \\
\hline
\end{tabular}

Legenda: M - masculino; F - feminino. Fonte: RHC - INCA e SigTap- SUS.

Na Tabela 2 observamos os resultados do custo-efetividade dos tratamentos de acordo como sexo dos pacientes, incluindo o custo (em reais), o custo incremental (diferença entre o custo do tratamento em questão com o de menor custo [Qt]), a efetividade, que é o inverso da razão de prevalência dos óbitos, a efetividade incremental, o RCEI (Razão de Custo-Efetividade Incremental) e o NMB (Net MonetaryBenefit). 
Tabela 2 - Distribuição dos tratamentos de acordo com o custo-efetividade dos tratamentos e o sexo dos pacientes.

\begin{tabular}{|c|c|c|c|c|c|c|c|}
\hline $\begin{array}{c}\text { Sexo/ } \\
\text { Tratamento }\end{array}$ & $\begin{array}{c}\text { Custo } \\
(R \$)\end{array}$ & $\begin{array}{c}\text { Custo } \\
\text { Incremental } \\
(R \$)\end{array}$ & Efetividade & $\begin{array}{c}\text { Efetividade } \\
\text { Incr }\end{array}$ & $R C E I$ & $N M B$ & $\begin{array}{c}\text { Custo/ } \\
\text { Efetividade }\end{array}$ \\
\hline \multicolumn{8}{|l|}{ Masculino } \\
\hline $\begin{array}{l}\text { Qt } \\
\text { QtRxt } \\
\text { QtTMO } \\
\text { TMO }\end{array}$ & $\begin{array}{c}11664 \\
14823 \\
74934,76 \\
63270,76\end{array}$ & $\begin{array}{c}0 \\
3159 \\
60111,76 \\
48447,76\end{array}$ & $\begin{array}{c}1 \\
1,759 \\
4,667 \\
0,663\end{array}$ & $\begin{array}{c}0 \\
0,759 \\
2,908 \\
-1,096\end{array}$ & $\begin{array}{c}0 \\
4162,05 \\
20671,17 \\
-44204,2\end{array}$ & $\begin{array}{c}-11664 \\
-14823 \\
-74934,8 \\
-63270,8\end{array}$ & $\begin{array}{c}11664 \\
8426,95 \\
16056,3 \\
95431,01\end{array}$ \\
\hline \multicolumn{8}{|l|}{ Feminino } \\
\hline $\begin{array}{l}\text { Qt } \\
\text { QtRxt } \\
\text { QtTMO } \\
\text { TMO }\end{array}$ & $\begin{array}{c}11664 \\
14823 \\
63270,76 \\
74934,76\end{array}$ & $\begin{array}{c}0 \\
3159 \\
48447,76 \\
60111,76\end{array}$ & $\begin{array}{c}1 \\
1,526 \\
0,424 \\
0,732\end{array}$ & $\begin{array}{c}0 \\
0,526 \\
-1,102 \\
-0,794\end{array}$ & $\begin{array}{c}0 \\
6005,703 \\
-43963,5 \\
-75707,5\end{array}$ & $\begin{array}{c}-11664 \\
-14823 \\
-63270,8 \\
-74934,8\end{array}$ & $\begin{array}{c}11664 \\
9713,63 \\
149223,5 \\
102369,9\end{array}$ \\
\hline
\end{tabular}

Fonte: Software TreeAge.

Para os pacientes do sexo masculino, o TMO não é custo-efetiva. Dessa forma, com o referido tratamento, o custo é maior e a sobrevida dos pacientes é menor. Por sua vez, embora possua alto custo incremental (R\$60.111,76), o tratamento com QtTMO em pacientes do sexo masculino apresenta efetividade muito superior $(4,667)$ às alternativas, tornando-se custo-efetiva. Nesse grupo de pacientes, a QtRxt, mesmo apresentando efetividade inferior, é a mais custo-efetiva por se tratar de uma estratégia com um custo menor (Tabela 2).

Nos pacientes do sexo feminino, à semelhança do observado para os pacientes do sexo masculino, a QtRxt se apresentou como modalidade de tratamento mais custo-efetiva. O TMO, por outro lado, demonstrou uma efetividade inferior, não sendo uma boa estratégia. Os resultados para QtTMO, ao contrário do constatado para os pacientes do sexo masculino, revelaram que esta é a modalidade de tratamento menos custo-efetiva nos indivíduos do sexo feminino, com uma efetividade incremental de ($1,102)$.

Os Gráficos 1 e 2 mostram as estratégias que obtiveram melhores resultados, tornando-se não dominadas e as que apresentaram resultados inferiores, expressas como dominadas. Observamos que o TMO sozinho não é uma alternativa viável, em ambos os sexos, pois custa muito e salva poucos pacientes. A Qt associada à TMO é o tratamento mais efetivo, e seu uso deve ser balanceado quanto à disponibilidade de recursos. 
Gráfico 1 - Análise de custo-efetividade para o sexo masculino.

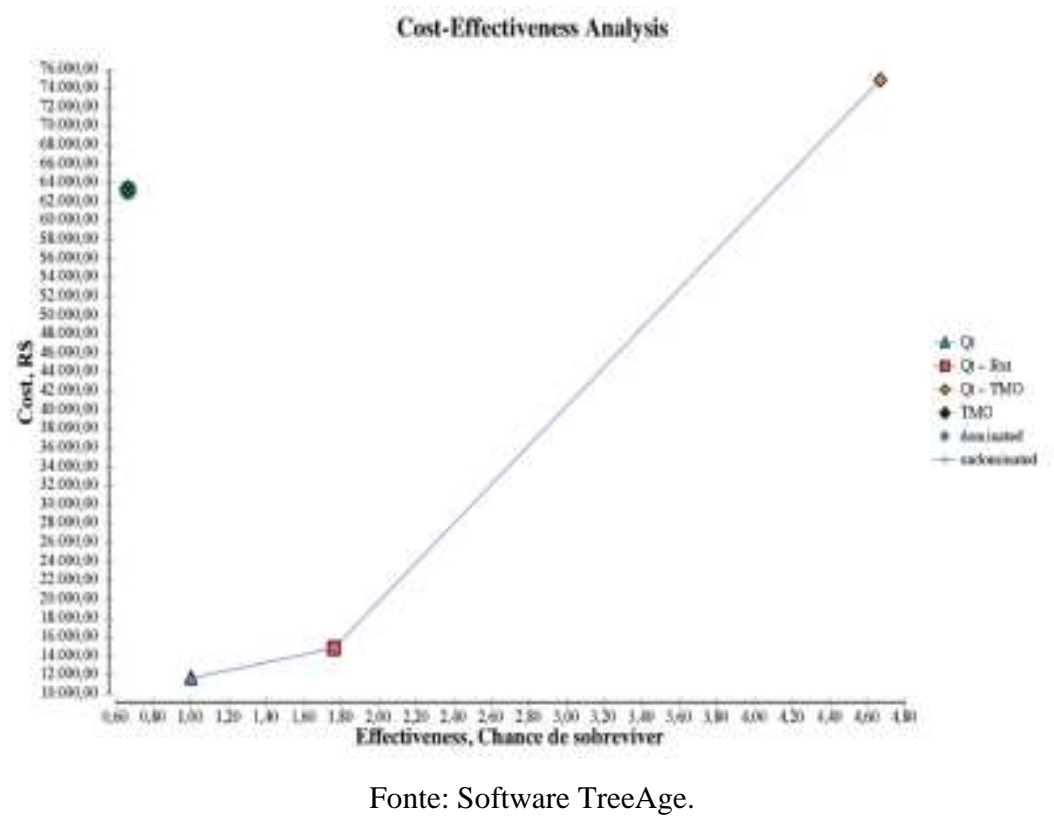

Gráfico 2 - Análise de custo-efetividade para o sexo feminino.

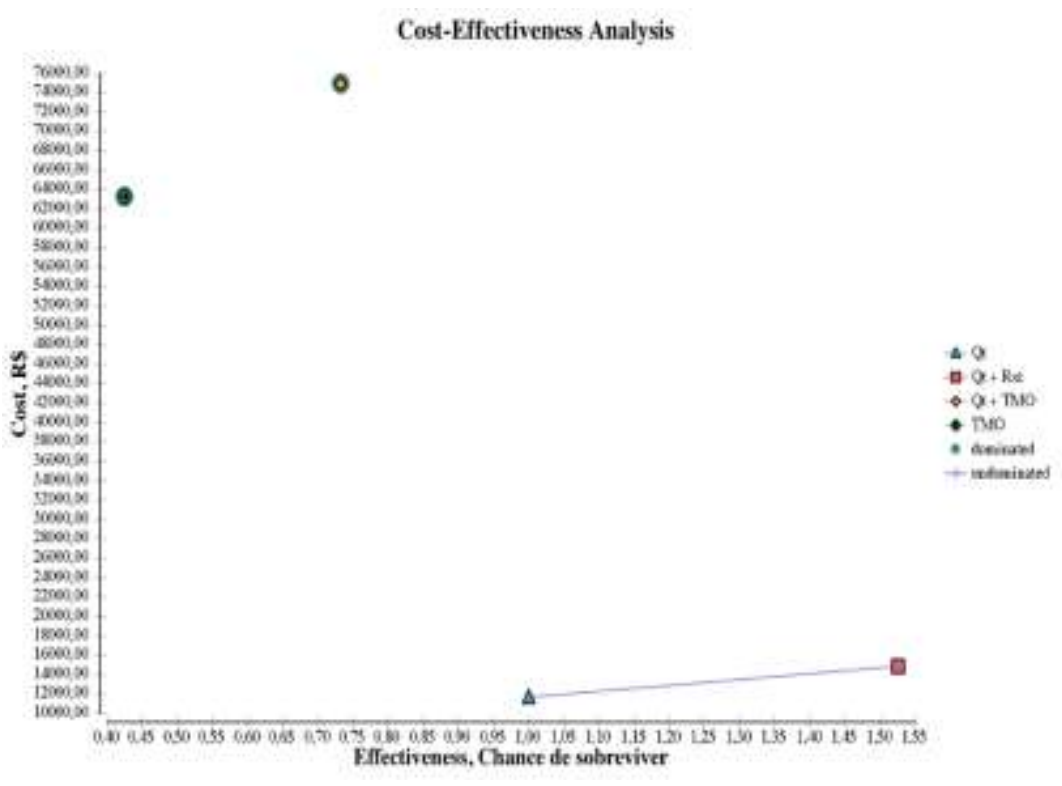

Fonte: Software TreeAge.

\section{Discussão}

A LLA acomete crianças e adolescentes, com prevalência de $75 \%$ na faixa etária de 2 a 5 anos. No entanto, a doença possui bom prognóstico, com uma sobrevida superior a 80\% em cinco anos (Santos, 2017; Cavalcante, Rosa, Torres, 2017; Silva, Silva, Cardoso, 2018). Não obstante, a investigação de abordagens terapêuticas custo-efetivas se faz necessário para reduzir a mortalidade dos indivíduos afetados e ampliar sua sobrevida.

O tratamento pode variar de acordo com o tipo de câncer (Santos, 2017). E este estudo demonstra também que, neste caso, a efetividade desses tratamentos varia quanto ao sexo. Talvez pela gravidade dos tumores ou grau de evolução dos casos que passaram por tratamento hospitalar. 
Os esquemas terapêuticos dependem do protocolo adotado em cada centro de especialização e pode envolver combinações complexas, com o uso de múltiplas drogas (Santos, 2017; Fung et al., 2019). Os custos desses tratamentos variam de acordo com a sua complexidade, incluindo a duração da quimioterapia, necessidade de cirurgia e necessidade de suporte e cuidado (Fung et al., 2019).

No Brasil, boa parte desses tratamentos são cobertos pelo sistema público de saúde, o que implica em um planejamento eficiente dos recursos. Assim, deve-se investir em estratégias que ampliem a chance de sobrevida dos pacientes. Evitando abordagens de TMO não associada à Qt, por exemplo, pois apresentam um custo muito alto e baixa efetividade.

No presente estudo, a Qt foi o tratamento mais prevalente para as leucemias linfocítica/linfóide/linfoblástica em ambos os sexos, mostrando uma boa taxa de remissão e estabilidade da doença, como também um bom custo-efetividade, revelando-se a estratégia mais barata. Tais achados corroboram a pesquisa realizada por Van Litsenburg et al. (2011), que avaliou o custoefetividade apenas da Qt no tratamento da LLA. De acordo com esses autores, independente do protocolo escolhido, a Qt se encontra dentro da faixa de custo-benefício aceitável para o tratamento da LLA.

O TMO, quando utilizado de forma isolada, mostrou efetividade relativamente baixa, em ambos os sexos, sendo inferior à da Qt, contrapondo-se às evidências encontradas em estudo realizado por Ashfaq, Yahaya e Hyde (2010). Conforme os resultados obtidos por esses autores, o transplante alogênico de células-tronco hematopoiéticas (CTH), de doadores irmãos, pode ser mais eficaz em comparação com a quimioterapia no tratamento da LLA em primeira remissão clínica. Por isso se faz necessário investigar mais a respeito do custo-efetividade dessa intervenção, ampliar o quantitativo de doadores, capacitar unidades hospitalares.

A avaliação da QtTMO para o sexo masculino, mostrou o melhor resultado da efetividade. Entretanto, para o sexo feminino, essa modalidade terapêutica não revelou bons resultados, tornando-se uma estratégia dominada. Essa combinação de tratamentos para a leucemia aguda pode acontecer quando são realizados os transplantes autólogos, utilizando altas doses de drogas quimioterápicas, seguidos de resgate com CTH. Tais terapias combinadas tem o objetivo de suplantar a resistência das células tumorais a doses convencionais de quimioterapia e melhorar a resposta ao tratamento (Barban, Simões, Moraes, 2020).

Avaliando o estudo retrospectivo com pacientes transplantados realizado por Morando, Mauad e Fortier (2010), percebemos que a incidência cumulativa de mortalidade relacionada ao transplante (MRT) foi significativamente superior em pacientes que realizaram transplante não aparentado, que tinham doença avançada no momento do transplante, com idades acima dos 10 anos, que desenvolveram doença do enxerto contra hospedeiro aguda e cujo transplante fora realizado com células provenientes de cordão umbilical. Na análise multivariada, por outro lado, o único fator estatisticamente significativo para MRT foi doença avançada no momento do transplante.

Como para esse estudo não temos o conhecimento acerca das condições dos pacientes no início do tratamento, nem do tipo de transplante que foi realizado para cada caso, podemos aferir apenas que o TMO isolado não se apresenta como uma alternativa viável para esse tipo de patologia, independente do sexo do paciente. Além disso, pode-se sugerir que a QtTMO não é uma alternativa custo-efetiva para pacientes femininos com LLA, mas consiste em uma ótima escolha para o tratamento de indivíduos do sexo masculino acometidos por essa neoplasia hematológica.

Em casos como esses, a identificação precoce é de extrema importância para se encontrar um doador compatível e melhorar o prognóstico desses pacientes. No entanto, embora alguns pacientes submetidos a transplante em primeira remissão apresentem bons resultados de sobrevida, há uma relação cuidadosa a se fazer quanto à sua necessidade, pois existe a possibilidade de recidiva nos primeiros 5 anos após o tratamento. Mesmo com o risco de recidiva, o TMO deve ser reservado à pacientes de alto risco ou com recidiva após a primeira remissão (Pinheiro et.al., 2019)

No presente estudo, o tratamento da LLA com QtRxt trouxe melhores resultados na relação custo-efetividade, tanto para o sexo masculino quanto para o feminino, tornando-o a melhor estratégia terapêutica para esse tipo de neoplasia em pacientes 
pediátricos. A Rxt pode ser realizada juntamente com a Qt, nas fases pré, per ou pós-operatória e se classifica como: radical ou curativa, quando se busca a cura total do tumor; remissiva, quando o objetivo é apenas redução tumoral; ou profilática, quando se busca a remissão de sintomas, tais como dor intensa, sangramento e compressão de órgãos (Oliveira, 2018).

Contudo, mesmo se tratando de uma estratégia com ótimos resultados e sendo bastante custo-efetiva, o tratamento com Rxt também traz alguns efeitos adversos para o paciente, como: mucosite, radiodermite, trismo, xerostomia e osteorradionecrose (Oliveira, 2018). Considerando a imunossupressão provocada pela Qt e os efeitos adversos da Rxt, a associação dessas terapias no tratamento da leucemia linfocítica/linfóide/linfoblástica poderá intensificar as complicações bucais e resultar em aumento importante da morbidade (Ferraz et al., 2018).

\section{Conclusão}

Dentre as formas de tratamento da LLA analisadas neste estudo, a QtRxt obteve os melhores resultados na sobrevida do paciente e a TMO, os piores, independente do sexo do paciente. Essa análise mostrou, por sua vez, diferentes cenários de acordo com o sexo do paciente, no qual a QtTMO apresentou-se como uma estratégia custo-efetiva para o sexo masculino, porém obteve resultados inferiores para o sexo feminino.

Contudo, mesmo com os resultados aqui obtidos, vale salientar que cada paciente deve ser avaliado individualmente para a escolha do tratamento ideal. Há a necessidade de mais estudos sobre o tratamento das leucemias na população infantojuvenil e seus custos e efetividades.

\section{Referências}

Angevine, P. D., \& Berven, S. (2014). Health economic studies: an introduction to cost-benefit, cost-effectiveness, and cost-utility analyses. Spine, 39(22 Suppl 1), S9-S15.

Arnaldi, A. P. (2019). Dissertação Análise do custo efetividade dos protocolos LLA 97 e GBTLI 99 para crianças em tratamento de leucemia linfocítica aguda. Universidade de São Paulo.

Ashfaq, K., Yahaya, I. \& Hyde, C. (2010). Clinical effectiveness and cost-effectiveness of stem cell transplantation in the management of acute leukaemia: a systematic review. NIHR Health Technology Assessment programme: Executive Summaries (NIHR Journals Library), 14(54), 1-141.

Barban, J. B., Simões, B. P. \& Moraes, B. D. G. D. C. (2020). Consenso Brasileiro de Nutrição em Transplante de Células-Tronco Hematopoiéticas: Adultos. Einstein (São Paulo), 18, 1-50.

Cavalcante, M. S., Rosa, I. S. S., \& Torres, F. (2017). Leucemia linfóide aguda e seus principais conceitos. Revista Científica da Faculdade de Educação e Meio Ambiente, 8(2), 151-164.

Ferraz, C. R. G., Lorena Sobrinho, J. E. D., Santos, J. P. A. \& Xavier, L. S. D. A. (2018). Monografia Manifestações orais em pacientes submetidos a quimioterapia e radioterapia: estratégias de controle e tratamento. Centro Universitário Tabosa de Almeida.

Fung, A., Horton, S., Zabih, V., Denburg, A., \& Gupta, S. (2019). Cost and cost-effectiveness of childhood cancer treatment in low-income and middle-income countries: a systematic review. BMJ global health, 4(5), 1-13.

INCA (2016). Incidência, mortalidade e morbidade hospitalar por câncer em crianças, adolescentes e adultos jovens no Brasil: informações dos registros de câncer e do sistema demortalidade. Instituto Nacional de Câncer José Alencar Gomes da Silva (INCA).

INCA (2019). Estimativa 2020: incidência de câncer no Brasil. Instituto Nacional de Câncer José Alencar Gomes da Silva (INCA).

INCA (2020). Câncer infantojuvenil. https://www.inca.gov.br/tipos-de-cancer/cancer-infantojuvenil.

Metayer, C., Milne, E., Clavel, J. et al. (2013). The childhood leukemia international consortium. Cancerepidemiology, 37(3), $336-347$.

Ministério da Saúde. (2017). Protocolo de diagnóstico precoce para oncologia pediátrica. http://bvsms.saude.gov.br/b vs/publicacoes/protocolo_diagnostico_precoce_cancer_pediatrico.pdf.

Morando, J., Mauad, M. A. \& Fortier, S. C. (2010). Transplante de células-tronco hematopoéticas em crianças e adolescentes com leucemia aguda: experiência de duas instituições Brasileiras. Revista Brasileira de Hematologia e Hemoterapia, 32(5), 350-357.

Moraz, G., Garcez, A. D. S., \& Assis, E.M. D. (2015). Estudos de custo-efetividade em saúde no Brasil: uma revisão sistemática. Ciência \& Saúde Coletiva, 20(10), 3211-3229. 
Research, Society and Development, v. 10, n. 14, e383101422056, 2021

(CC BY 4.0) | ISSN 2525-3409 | DOI: http://dx.doi.org/10.33448/rsd-v10i14.22056

Oliveira, F. A. S. D. (2018). Monografia Análise alteração de índices de hemograma de pacientes com leucemia linfóide crônica: uma revisão. Universidade Federal de Campina Grande.

Pinheiro, D. M., Silva, M. R., Silva, J. R. \& de Melo Diniz, R. T. (2019). Leucemia Linfóide Aguda Tipo B: Relato de um Caso sem Transplante de Medula Óssea. Anais do Seminário Científico do UNIFACIG 2019, (5), 1-5.

Santos, M. H. S. (2017). Monografia Mortalidade e fatores associados ao óbito em crianças e adolescentes com leucemia linfóide aguda tratadas em um serviço de referência no nordeste do Brasil. Universidade Federal de Sergipe.

Silva, C. D. S., Sousa, R. R., \& Cardoso, R. O. (2018). Leucemia linfocítica aguda na infância e suas complicações. Revista de Iniciação Científica e Extensão, $1(2), 109-113$.

Silva, E. N. D., Silva, M. T., \& Pereira, M. G. (2016). Modelos analíticos em estudos de avaliação econômica. Epidemiologia e Serviços de Saúde, 25(4), 855858.

Silva, J. (2019). Avaliação de Custo-efetividade nas terapias oncológicas, emprego de modelos de Markov: Uma revisão integrativa da literatura. REAS/EJCH, 11(15), 1-16.

Van Litsenburg, R. R., Uyl-de Groot, C. A., Raat, H., Kaspers, G. J., \& Gemke, R. J. (2011). Cost-effectiveness of treatment of childhood acute lymphoblastic leukemia with chemotherapy only: the influence of new medication and diagnostic technology. Pediatricblood \&câncer, 57(6), 1005-1010.

Viana, S. S., Lima, L. M. M. R., Nascimento, J. B. et al. (2015) Secular trends and predictors of mortality in acute lymphoblastic leukemia for children of low socioeconomic level in Northeast Brazil. Leukemiaresearch, 39(10), 1060-1065. 